\title{
Feshbach Resonance Cooling of Trapped Atom Pairs
}

\author{
Josh W. Dunn, ${ }^{1}$ D. Blume,${ }^{2}$ Bogdan Borca,${ }^{1}$ B. E. Granger,${ }^{3}$ and Chris H. Greene ${ }^{1}$ \\ ${ }^{1}$ Department of Physics and JILA, University of Colorado, Boulder, Colorado 80309-0440 \\ ${ }^{2}$ Department of Physics, Washington State University, Pullman, Washington 99164-2814 \\ ${ }^{3}$ Department of Physics, Santa Clara University, Santa Clara, CA 95053
}

(Dated: November 15, 2018)

\begin{abstract}
Spectroscopic studies of few-body systems at ultracold temperatures provide valuable information that often cannot be extracted in a hot environment. Considering a pair of atoms, we propose a cooling mechanism that makes use of a scattering Feshbach resonance. Application of a series of time-dependent magnetic field ramps results in the situation in which either zero, one, or two atoms remain trapped. If two atoms remain in the trap after the field ramps are completed, then they have been cooled. Application of the proposed cooling mechanism to optical traps or lattices is considered.

PACS numbers: 32.80.Pj, 03.75.-b, 34.50.-s
\end{abstract}

A Feshbach resonance 1, 2, 3] occurs for two atoms when their collision energy becomes degenerate with a vbound state in a closed collision channel, producing brief transitions into and out of this state. In recent years, these resonances have been used extensively to control the interaction strength in dilute atomic gases [4, [5, 6]. Here, we utilize some of the unique characteristics of a Feshbach resonance to develop a cooling mechanism that is applicable to two externally-confined atoms. Application of a series of field ramps (i.e., cooling cycles) leads to a cooled atom pair (an atom pair with reduced internal energy), provided that the atom pair remains trapped.

In the following, we first develop the basic mechanism of the Feshbach resonance cooling process. The feasibility and effectiveness of the proposed scheme are then illustrated through an application to a realistic system of two atoms in a trap. Finally, possible applications to optical traps are discussed.

The concept of Feshbach resonance cooling grows out of the observation that the quantum-mechanical energy levels of two atoms in a harmonic trap shift by an energy corresponding to approximately two trap quanta, as a control parameter is swept in one direction across the resonance. Throughout this article, we refer to this control parameter as the magnetic field $B$ used to manipulate the atom-atom scattering length $a$ in the vicinity of a pole. In other contexts, the shift of the energy levels could be introduced by varying the detuning of an off-resonant dressing laser, or by varying an electric field strength. The ideas presented here in terms of the control parameter $B$ can be straightforwardly extended to those other contexts.

The Schrödinger equation for two interacting identical mass $m$ atoms under spherical harmonic confinement with trapping frequency $\nu$ decouples into two equations: one involving the three relative coordinates of the pair, and another involving the three center-of-mass (CM) coordinates [7, [8]. We consider the Schrödinger equation in the relative coordinate for two trapped atoms interacting through a central potential and assume for the time being that the center of mass coordinate is translationally cold. Accounting for an applied external magnetic field $B$ through a $B$-dependent quantum defect $\beta_{E l}(B)$, the energies $E_{n l}(B)$ associated with the relative motion of an atom pair are given by [ $[$ ]

$$
E_{n l}(B)=\left(2 n-2 \beta_{E l}(B)+l+3 / 2\right) \hbar \omega,
$$

where $\omega=2 \pi \nu$. Here, the quantum defect $\beta_{E l}(B)$ depends strongly on the relative orbital angular momentum $l$ of the pair, while it depends only weakly on the radial oscillator quantum number $n$. The dependence of $\beta_{E l}(B)$ on the energy is weak on the scale of an oscillator quantum, i.e., $\left|\mathrm{d} \beta_{E l}(B) / \mathrm{d} E_{n l}\right| \ll 1 / \hbar \omega$.

As will become clear later, the quantum defect for one relative partial wave $l$ for an atom pair, e.g., the $s$-wave, $p$-wave, or $d$-wave, must rise by unity across the energy range $k_{\mathrm{B}} \Delta T$ of interest, and across the accessible range of the control parameter, $\Delta B$. In fact, this variation of $\beta_{E l}(B)$ by unity corresponds to the Feshbach resonance, which causes the scattering phaseshift to rise by $\pi$. A simple closed-form expression exists for $\beta_{E l}$ [8, 9], which simplifies at energies higher than a few trap quanta to $\beta_{E l}(B) \approx \arctan \left(\frac{a\left(E_{n l}, B\right) \hbar \omega}{2 L_{\mathrm{osc}} E_{n l} \sqrt{e}}\right)$ where $a\left(E_{n l}, B\right)$ is the energy- and field-dependent scattering length and $L_{\mathrm{osc}}=\sqrt{\hbar /(\mu \omega)}$ with $\mu=m / 2$ denotes the characteristic oscillator length.

In this paper, we focus on an $s$-wave resonance, though this formalism can be readily extended to higher partial wave resonances. When an $s$-wave Feshbach resonance occurs, the limiting low-energy scattering phaseshift is proportional to the wavenumber $k=\left(2 \mu E / \hbar^{2}\right)^{1 / 2}$. Omitting the subscript $l$, the $E$ - and $B$-dependent scattering length is then given by

$$
a\left(E_{n}, B\right)=a_{\mathrm{bg}}+\frac{\Gamma_{E} \sqrt{\hbar^{2} /\left(8 \mu E_{n}\right)}}{E_{n}+\left(B-B_{\mathrm{res}}\right) E_{\mathrm{res}}^{\prime}(B)},
$$

where $a_{\mathrm{bg}}$ is the background scattering length. At the magnetic field strength $B_{\text {res }}$ of the resonance a zeroenergy bound state occurs. The resonance width in energy $\Gamma_{E}$ is related to the width in the control parameter 


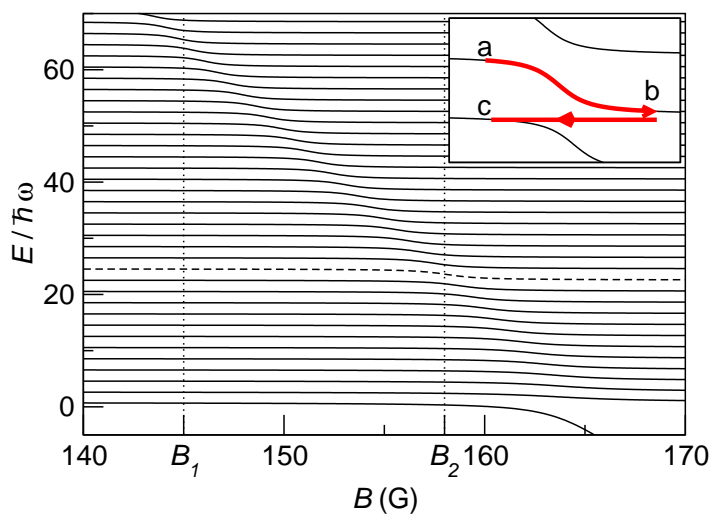

FIG. 1: (Color online) Energy levels $E_{n}$ for the relative coordinate of a harmonically trapped ${ }^{85} \mathrm{Rb}$ atom pair near the $B_{0} \approx 155.2 \mathrm{G}$ Feshbach resonance, as a function of the magnetic field $B$. A rather large trapping frequency of $\nu=1 \mathrm{MHz}$ is used in order to clarify the field dependence of the energy levels. Cooling is performed by ramping the magnetic field $B$ slowly from $B_{1}$ to $B_{2}$ and then quickly back to $B_{1}$. A more realistic ramp would likely encompass more level curves (i.e., cover a larger field range). The state which undergoes a shift for $B=B_{2}$ (which we will label as $n=Q$ later) is indicated by a dashed line. Ideal cooling is described diagramatically in the inset (same axes), where population transfer from point $a$ to point $b$ occurs during the slow field ramp and from $b$ to c during the fast ramp. See the text for details.

$\Delta$ by $\Gamma_{E}=2 k a_{\mathrm{bg}} E_{\mathrm{res}}^{\prime}(B) \Delta$, where $E_{\mathrm{res}}^{\prime}$ denotes the rate at which the resonance energy $E_{\text {res }}$ varies with the control parameter [10]. Figure[illustrates the characteristic $s$-wave energy levels $E_{n}$ appropriate for the relative motion of two atoms in a spherical harmonic oscillator trap, as functions of the applied magnetic field $B$ for a magnetic Feshbach resonance in ${ }^{85} \mathrm{Rb}(2,-2)+{ }^{85} \mathrm{Rb}(2,-2)$. In Fig. 1 the parameters adopted are $B_{\text {res }}=155.2 \mathrm{G}$, $E_{\text {res }}^{\prime}=-3.5 \mathrm{MHz} / \mathrm{G}, \Gamma_{B}=10 \mathrm{G}$, and $a_{\mathrm{bg}}=-380 a_{0}$, where $a_{0}$ is the Bohr radius.

Feshbach resonance cooling entails ramping the magnetic field through the region where the energy levels shift by $\approx 2 \hbar \omega$. Figure $\square$ denotes the initial $B$-field by $B_{1}$. For an atom pair taken from a source of atoms with temperature $T$, the probability of the pair being in the energy eigenstate $\left|n\left(B_{1}\right)\right\rangle$, and hence, of having the energy $E_{n}\left(B_{1}\right)$, is given by the corresponding Boltzmann factor. By ramping the magnetic field from $B_{1}$ to $B_{2}$ (also shown in the inset of Fig. 1), sufficiently slowly to be adiabatic, the population of each energy level remains unchanged, while the energy level itself is reduced compared to its value at $B_{1}, E_{n}\left(B_{2}\right) \approx E_{n}\left(B_{1}\right)-2 \hbar \omega$; the atom pair has lost energy. To further reduce the energy of the two atoms, another field ramp has to be applied. In order to do this, it is necessary to return the magnetic field to $B_{1}$ without adding the energy back that has been just removed. A fast, nonadiabatic change of the magnetic field from $B_{2}$ to $B_{1}$ ensures this, since this change simply projects the eigenstate $\left|n\left(B_{2}\right)\right\rangle$ onto the eigenstate $\left|n\left(B_{1}\right)\right\rangle$. Note that this proposed energy reduction (cooling) comes at the expense of an energy gain of a single level (or a few levels, see below for a detailed discussion).

To model the effects of the magnetic field ramps, we have developed a two-channel Feshbach resonance model, based on the single-channel model described in Ref. 11]. Both of these models describe a two-atom Feshbach resonance for a harmonic trap, and utilize a zero-range potential to describe the interaction between the two atoms. The two-channel model has the advantage of allowing for a field-dependent resonance state. In the two channels, the $s$-wave radial solutions for the relative coordinate $r$ of the atom pair satisfy the equations

$$
\begin{gathered}
\left(-\frac{\hbar^{2}}{2 \mu} \frac{\mathrm{d}^{2}}{\mathrm{~d} r^{2}}+\frac{1}{2} \mu \omega r^{2}\right) u_{1}(r)=E u_{1}(r) \\
\left(-\frac{\hbar^{2}}{2 \mu} \frac{\mathrm{d}^{2}}{\mathrm{~d} r^{2}}+\frac{1}{2} \mu \omega r^{2}\right) u_{2}(r)=(E-\varepsilon) u_{2}(r),
\end{gathered}
$$

where $\varepsilon$ is the energy shift of the second channel from the first channel. The zero-range potential imposes a boundary condition at the origin, which is parameterized as

$$
\frac{\mathrm{d}}{\mathrm{d} r}\left(\begin{array}{l}
u_{1}(r) \\
u_{2}(r)
\end{array}\right)_{r=0}=\left(\begin{array}{cc}
-1 / a_{1} & \beta \\
\beta & -1 / a_{2}
\end{array}\right)\left(\begin{array}{l}
u_{1}(r) \\
u_{2}(r)
\end{array}\right)_{r=0} .
$$

A quantum-defect-theory treatment, similar to Ref. 11], can then be applied. The scattering length predicted by this model (when $\omega \rightarrow 0$ ) is

$$
a(E, B)=\left(\frac{1}{a_{1}}+\frac{|\beta|^{2}}{\sqrt{2 \mu \varepsilon(B) / \hbar^{2}-2 \mu E / \hbar^{2}}-1 / a_{2}}\right)^{-1},
$$

which can be compared to the measured scattering length to determine the values of the parameters $a_{1}, a_{2}$, and $\beta$. The parameters also affect the magnetic-field dependence of the adiabatic energy states, and their adjustment is able to provide satisfactory agreement with experimental data in the regions of interest to us. E.g., for ${ }^{85} \mathrm{Rb}$, we find $a_{1}=-435 a_{0}, a_{2}=1.485 a_{0}$, and $\beta=0.0011618 a_{0}^{-1}$. Simulations can then be performed by specifying an initial state of the system and numerically propagating the Schrödinger equation.

The simulations reveal the effect of the adiabatic and nonadiabatic field ramps: Assume first that the atom pair is in a pure state at $B=B_{1}$. As expected, the adiabatic field ramp ( $B_{1}$ to $B_{2}$ in Fig. (1) decreases the energy of the atom pair irrespective of the initial eigenstate chosen. A nonadiabatic ramp ( $B_{2}$ to $B_{1}$ in Fig. 11) causes a state at $B_{2}$ that is not degenerate with the resonance state to project onto a state at $B_{1}$, with approximately the same energy as the initial state at $B_{2}$. However, if the pair is initially in the state at $B_{2}$ that is degenerate with the resonance state, the fast ramp results in a strong projection onto the resonance state. In this case, the atom pair gains energy since the resonance state at $B_{1}$ has a higher energy than the initial state at $B_{2}$. 
We now generalize our scheme to the more practical situation of a mixed initial state, and show how removal of hot atoms leads to an intriguing cooling scheme. For a mixed state, the occupation probability of a pair level with energy $E_{n}$ in the relative motion is determined in terms of a Boltzmann factor by $e^{-E_{n} / \tau} / Z(\tau)$ with $\tau=k_{\mathrm{B}} T$, where $k_{\mathrm{B}}$ is Boltzmann's constant, $Z(\tau)$ is the (relative) partition function, and $T$ is the temperature of the source of the two atoms. Based on the results of field ramps for pure states discussed above, we see that application of a cooling cycle (slow ramp from $B_{1}$ to $B_{2}$ plus fast ramp back to $B_{1}$ ) for a mixed state will do two things: (a) decrease by $2 \hbar \omega$ the energy of the population in states which undergo a full energy shift between $B_{1}$ and $B_{2}$, and (b) increase the energy of the population in the state that is degenerate with the resonance (i.e., undergoing an energy shift) at $B_{2}$ by moving it to the state (or states) degenerate with the resonance at $B_{1}$. We will denote this state from which the heated population originates as $n=Q$.

Figure 2 illustrates the effect of a single cooling cycle on a mixed state, using the same Feshbach resonance as shown in Fig. 1] The black line represents an initial $s$-wave probability distribution for the states associated with the relative coordinate of an atom pair in a harmonic trap with $\nu=1 \mathrm{MHz}$ and source temperature $T=1 \mathrm{mK}$. The red line represents the same probability distribution after application of a slow and a fast magnetic field ramp, for $Q=10$. Application of one cooling cycle moves the population of the state $Q$ to states with much higher energy, here $n \approx 85$, evidenced by the spike in Fig. 2 At the same time, the field ramps move the population of each state with $n>Q$ to the next-lowest state, which has $\approx 2 \hbar \omega$ less energy. Our numerical simulations indicate that, on average, the net energy of the system is increased after application of one cooling cycle. The few cases where the pair gains a large amount of energy overcomes the many cases where the pair looses a small amount of energy. This behavior is expected, since Ketterle and Pritchard [12] in 1992 pointed out the impossibility of creating a cooling scheme relying solely on time-dependent potentials.

An estimate of the efficiency of cooling can be made by assuming that the population of the state $Q$ is removed from the trap, while the population of all states with $n>Q$ are moved to the next-lowest state, that is, to states with $n-1$. This assumes that the range of the field ramps is such that the heated fraction $(n=Q)$ ends up at an energy corresponding to negligible thermal population (this is the case in Fig. 22), and that all population above a specified energy can be removed. If we approximate the level energies at $B=B_{1}$ by $E_{n}\left(B_{1}\right) \approx 2 n \hbar \omega$, the probability to remove an atom pair during a cooling cycle is

$$
P_{\mathrm{rem}}(Q, \tau)=\frac{e^{-2 \hbar \omega Q / \tau}}{Z(\tau)}
$$

The average energy decrease in a cooling cycle is due to

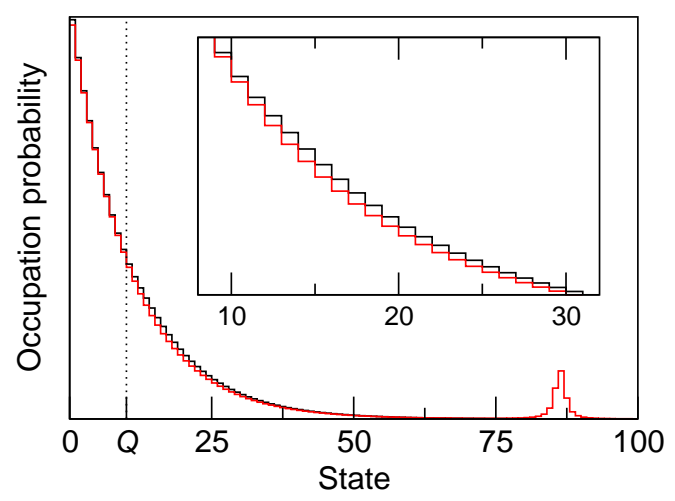

FIG. 2: (Color online) Illustration of the effect of a single Feshbach resonance cooling cycle for $T=1 \mathrm{mK}$ and $\nu=1 \mathrm{MHz}$. The black (red) line represents the population distribution before (after) application of one slow and one fast magnetic field ramp. The state $Q$ (here $Q=10$ ) is indicated. Population from $n=Q$ and nearby states is moved to higher states with $n \approx 85$. Population initially in a state with $n>Q$ is moved to the next-lowest state (see the inset close-up).

the energy of the $n \approx Q$ population removed from the trap, plus the energy loss for states $n>Q$ :

$$
\begin{aligned}
& \Delta E(Q, \tau)=\left(2 \hbar \omega Q+\left\langle E_{\mathrm{CM}}\right\rangle\right) \frac{e^{-2 \hbar \omega Q / \tau}}{Z(\tau)} \\
&+\sum_{n=Q+1}^{\infty} 2 \hbar \omega \frac{e^{-2 \hbar \omega n / \tau}}{Z(\tau)} .
\end{aligned}
$$

Noting that $\left\langle E_{\mathrm{CM}}\right\rangle=3 \tau$ (since $\left\langle E_{\mathrm{tot}}\right\rangle=3 \tau$ for a single atom in a harmonic trap), and with $\sum_{n=Q+1}^{\infty} e^{-2 \hbar \omega n / \tau} \approx$ $e^{-2 \hbar \omega Q / \tau} \tau / 2 \hbar \omega$, Eq. (8) becomes

$$
\Delta E(Q, \tau)=\frac{e^{-2 \hbar \omega Q / \tau}}{Z(\tau)}(2 \hbar \omega Q+4 \tau) .
$$

The energy efficiency $E_{\text {eff }}$, defined as the amount of energy removed per atom removed, is then given by

$$
E_{\text {eff }}(Q, \tau)=2 \hbar \omega Q+4 \tau \text {. }
$$

Since $Q$ determines the efficiency of the cooling process, it is referred to as the cooling parameter. Results from our numerical model indicate that Eq. (10) provides a good estimate of the efficiency.

The time scale for one cooling cycle is determined by the speed of the adiabatic field ramp. This speed in turn is determined by the strength of the coupling between the resonance state and the trap states. The smaller the coupling for an avoided crossing, the slower is the field ramp required to maintain adiabaticity. The coupling between the resonance state and the trap states is related to the resonance width parameter $\Gamma_{E}$, which can be used in a Landau-Zener estimate of the transition probability [10],

$$
P_{\mathrm{tr}} \cong \exp \left(-\frac{2}{|\mathrm{~d} B / \mathrm{d} t|} \frac{\omega \Gamma_{E}}{|\mathrm{~d} E / \mathrm{d} B|}\right)
$$




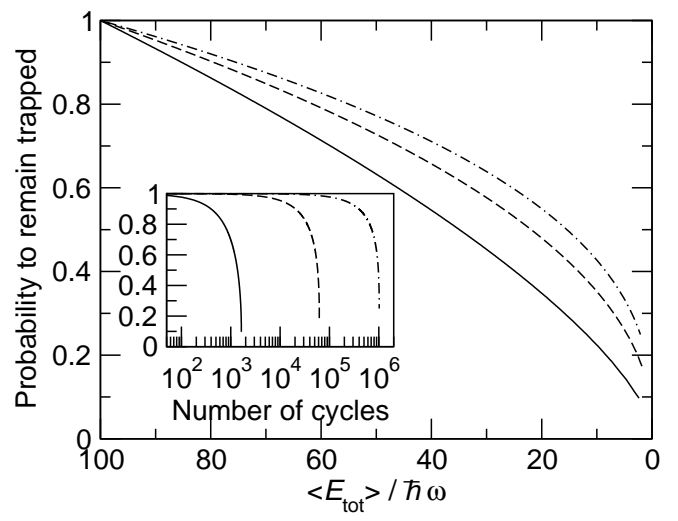

FIG. 3: Probability that a pair of atoms remains trapped vs. the average total kinetic energy of the two atoms in oscillator units (note that $k_{\mathrm{B}} T=\left\langle E_{\text {tot }}\right\rangle / 6$ for two harmonically trapped atoms). Three different cooling parameters are used: $2 \hbar \omega Q=5 \tau$ (solid line), $9 \tau$ (dashed line), and $12 \tau$ (dotdashed line). It is assumed that rethermalization occurs between cooling cycles (see text). Inset: probability to remain trapped vs. the number of cooling cycles for the same three cooling parameters.

Motivated by the possibility of experimentally trapping a small, deterministic number of atoms [13], we now explore the experimental feasibility of our cooling scheme. A Feshbach resonance cooling experiment involves a sequence of cooling cycles. As discussed above, a single experiment could result in a heated atom pair, which in turn would be lost from the trap. To end up with a cooled atom pair, multiple ramp cycles are required. To see the effect of cooling, Eqs. (77) and (8) can be iterated. For a variety of cooling efficiency parameters $Q$, Fig. 3 shows the probability for an atom pair to remain trapped vs. the average total kinetic energy (the energy of both the relative and the CM degrees of freedom) of the two atoms in oscillator units. Included in this calculation is the probability that the atom pair is in an $s$-wave state to begin with, because the field ramp has no effect on other partial waves. We assume that rethermalization occurs between cooling cycles, which could be ensured by, for example, introducing a slight anharmonicity into the trapping potential.

To be more specific, we consider a crossed-beam op- tical dipole trap [14] which offers a good blend of large trap frequency (for a large $s$-wave fraction), isotropy, and anharmonicity (for rethermalization between the relative and CM degrees of freedom). Assuming the dipole trap has an average frequency of $\nu=10 \mathrm{kHz}$ and contains two atoms taken from a source with temperature $T=8 \mu \mathrm{K}$ $\left(\left\langle E_{\text {tot }}\right\rangle / \hbar \omega=100\right)$, we see from Fig. 3] solid line, that a temperature of $0.16 \mu \mathrm{K}\left(\left\langle E_{\text {tot }}\right\rangle / \hbar \omega=2\right.$, both atoms in the ground state) could be reached $10 \%$ of the time by performing less than 20,000 cooling cycles. For a range in magnetic field for the ramps of $\Delta B \approx 1 \mathrm{G}$, and using Eq. (11) with $P_{\mathrm{tr}}=0.1$, we see that such a series of field ramps could take place in under $1 \mathrm{~s}$. A pertubative calculation accounting for the trap anharmonicity (see Ref. 15] for details of a similar treatment) indicates that rethermalization between the relative and CM degrees of freedom should occur on a time scale comparable to a single ramp time for a crossed-beam dipole trap. This will ensure that the relative $s$-wave distribution will rethermalize with each ramp and that the cooling of the relative coordinate will also cool the CM coordinate (both of which we have assumed up to this point).

Another possibility would be to apply our cooling scheme to atom pairs trapped in an optical lattice. In this case, field ramps could be performed on the lattice ensemble of atom pairs, with a certain percentage of sites resulting in cooled pairs, while other sites will have either zero atoms or one (uncooled) atom. It may also be possible to prepare the optical lattice by some other means to have a high probability of exactly double occupancy at each lattice cite (see for example Ref. 16|). From such an initial state, a Feshbach resonance cooling scheme could be used to efficiently cool atom pairs to low-lying trap states.

In summary, we have proposed a novel cooling scheme that makes use of Feshbach resonances. We have also shown that such a cooling scheme may be experimentally feasible, although it may not be competitive with proven cooling methods like evaporation. At the same time, plenty of room for exploration remains. Extension to atom clouds might also be possible.

This work was supported in part by the National Science Foundation. We thank M. Holland, C. Wieman, E. Cornell, P. Julienne, D. Jin, and M. Baertschy for helpful discussions.
[1] H. Feshbach, Theoretical Nuclear Physics (Wiley, New York, NY, 1992).

[2] W. C. Stwalley, Phys. Rev. Lett. 37, 1628 (1976).

[3] E. Tiesinga, B. J. Verhaar, and H. T. C. Stoof, Phys. Rev. A 47, 4114 (1993).

[4] S. Inouye, M. R. Andrews, J. Stenger, H.-J. Miesner, D. M. Stamper-Kurn, and W. Ketterle, Nature 392, 151 (1998).

[5] P. Courteille, R. S. Freeland, D. J. Heinzen, F. A. van
Abeelen, and B. J. Verhaar, Phys. Rev. Lett. 81, 69 (1998).

[6] J. L. Roberts, N. R. Claussen, J. P. Burke, Jr., C. H. Greene, E. A. Cornell, and C. E. Wieman, Phys. Rev. Lett. 81, 5109 (1998).

[7] T. Busch, B.-G. Englert, K. Rzaz̧ėwski, and M. Wilkins, Found. Phys. 28, 549 (1998).

[8] D. Blume and C. H. Greene, Phys. Rev. A 65, 043613 (2002). 
[9] E. L. Bolda, E. Tiesinga, and P. S. Julienne, Phys. Rev. A 66, 013403 (2002).

[10] F. H. Mies, E. Tiesinga, and P. S. Julienne, Phys. Rev. A 61, 022721 (2000).

[11] B. Borca, D. Blume, and C. H. Greene, New J. Phys. 5, 111.1 (2003).

[12] W. Ketterle and D. E. Pritchard, Phys. Rev. A 46, 4051 (1992).

[13] D. Frese, B. Ueberholz, S. Kuhr, W. Alt, D. Schrader,
V. Gomer, and D. Meschede, Phys. Rev. Lett. 85, 3777 (2000).

[14] M. Barrett, J. Sauer, and M. S. Chapman, Phys. Rev. Lett. 87, 010404 (2001).

[15] E. L. Bolda, E. Tiesinga, and P. S. Julienne, to be published (2004).

[16] P. Rabl, A. J. Daley, P. O. Fedichev, J. I. Cirac, and P. Zoller, Phys. Rev. Lett. 91, 110403 (2003). 